\title{
Lymphatic mimicry in maternal endothelial cells promotes placental spiral artery remodeling
}

\author{
John B. Pawlak, ${ }^{1}$ László Bálint, ${ }^{2,3}$ Lillian Lim,, ${ }^{4}$ Wanshu Ma, ${ }^{5}$ Reema B. Davis, ${ }^{1}$ Zoltán Benyó, ${ }^{6}$ Michael J. Soares, ${ }^{7,8}$ Guillermo Oliver, ${ }^{5}$ \\ Mark L. Kahn, ${ }^{4}$ Zoltán Jakus, ${ }^{2,3}$ and Kathleen M. Caron ${ }^{1}$ \\ 'Department of Cell Biology and Physiology, University of North Carolina, Chapel Hill, North Carolina, USA. Department of Physiology, Semmelweis University School of Medicine, Budapest, Hungary. \\ ${ }^{3}$ MTA-SE "Lendület" Lymphatic Physiology Research Group of the Hungarian Academy of Sciences and the Semmelweis University, Budapest, Hungary. ${ }^{4}$ Department of Medicine and Cardiovascular \\ Institute, University of Pennsylvania, Philadelphia, Pennsylvania, USA. ${ }^{5}$ Northwestern University, Feinberg School of Medicine, Chicago, Illinois, USA. Institute of Clinical Experimental Research, Semmelweis \\ University, Budapest, Hungary. 'Department of Pathology and Laboratory Medicine, University of Kansas Medical Center, Kansas City, Kansas, USA. ${ }^{8}$ Center for Perinatal Research, Children's Research \\ Institute, Children's Mercy, Kansas City, Missouri, USA.
}

\begin{abstract}
Molecular heterogeneity of endothelial cells underlies their highly specialized functions during changing physiological conditions within diverse vascular beds. For example, placental spiral arteries (SAs) undergo remarkable remodeling to meet the ever-growing demands of the fetus - a process which is deficient in preeclampsia. The extent to which maternal endothelial cells coordinate with immune cells and pregnancy hormones to promote SA remodeling remains largely unknown. Here we found that remodeled SAs expressed the lymphatic markers PROX1, LYVE1, and VEGFR3, mimicking lymphatic identity. Uterine natural killer (UNK) cells, which are required for SA remodeling and secrete VECFC, were both sufficient and necessary for VEGFR3 activation in vitro and in mice lacking uNK cells, respectively. Using Flt4 ${ }^{\text {chy/+ }}$ mice with kinase inactive VEGFR3 and Vegff ${ }^{f / f l}$ Vav1-Cre mice, we demonstrated that SA remodeling required VEGFR3 signaling, and that disrupted maternal VEGFR3 signaling contributed to late-gestation fetal growth restriction. Collectively, we identified a novel instance of lymphatic mimicry by which maternal endothelial cells promote SA remodeling, furthering our understanding of the vascular heterogeneity employed for the mitigation of pregnancy complications such as fetal growth restriction and preeclampsia.
\end{abstract}

\section{Introduction}

Endothelial cells (ECs) lining the vasculature display a large degree of phenotypic variation and molecular heterogeneity, reflecting the vastly different environmental milieus and functions of target end organs $(1,2)$. Homeostatic maintenance of a differentiated state within distinct organ environments is, by necessity, an active process that requires consistent activation or suppression of key signaling pathways. In contrast, physiological changes or disease conditions can disrupt these pathways, resulting in the loss of a differentiated state. Endothelial to mesenchymal transition and endothelial to hematopoietic transition remain striking examples of the extent to which endothelial cells may be altered from their formally differentiated, homeostatic states.

Intraendothelial transitions also occur, as exemplified by in vivo deletion of the lymphatic-fating nuclear receptor Prox1 in mouse lymphatic endothelial cells (LECs), resulting in a reduction of LEC identity with a concomitant enhancement of blood endothelial cell (BEC) identity (3). Inversely, BEC-specific PROX1 ectopic expression upregulates lymphatic genes while downregulating BEC-specific genes (4). Indeed, some structures exhibit an identity that is hybrid of both blood and lymphatic markers, such as the

Conflict of interest: The authors have declared that no conflict of interest exists. Copyright: (5) 2019, American Society for Clinical Investigation.

Submitted: March 12, 2018; Accepted: August 8, 2019; Published: October 14, 2019.

Reference information: J Clin Invest. 2019;129(11):4912-4921.

https://doi.org/10.1172/JCl120446
Schlemm's canal of the eye or the ascending vasa recta of the kidney, to ultimately underlie their highly specialized functions (5-7). It is likely that other hybrid vessels exist, but our understanding of the molecular markers and regulators of this organ- and vessel-specific endothelial plasticity remains limited.

One vessel that exhibits a high degree of plasticity is found in the specialized vascular bed of the placenta. Spiral arteries (SAs) of the maternal decidua dynamically regulate blood flow into the placenta to meet the ever-evolving nutritional and oxygenation needs of a growing fetus. During early to mid-gestation, SAs undergo remodeling characterized by luminal expansion facilitated by a combination of endothelial proliferation, degradation of extracellular matrix, and loss of smooth muscle coverage $(8,9)$. In humans, poor or failed spiral artery remodeling (SAR) is associated with preeclampsia, a potentially fatal hypertensive disease that occurs in $2 \%-8 \%$ of pregnancies, often causing fetal growth restriction and long-term health complications for both mother and fetus (10-12). Thus, there is great interest in elucidating the pregnancy-induced factors that serve as molecular determinants of SAR, with a particular focus on the crosstalk between SA endothelial cells and locally secreted trophoblast- and immune-cell factors.

Several studies have correlated an endothelial transition of SAs from arterial to venous fate during SAR, as evidenced by changes in the receptor tyrosine kinase family of ephrin receptors (13), which also have critical functions in lymphatic vessels (14-16). Moreover, in the early mouse implantation site, the vascular fold anlage of SAs also express high levels of VEGFR3 and 

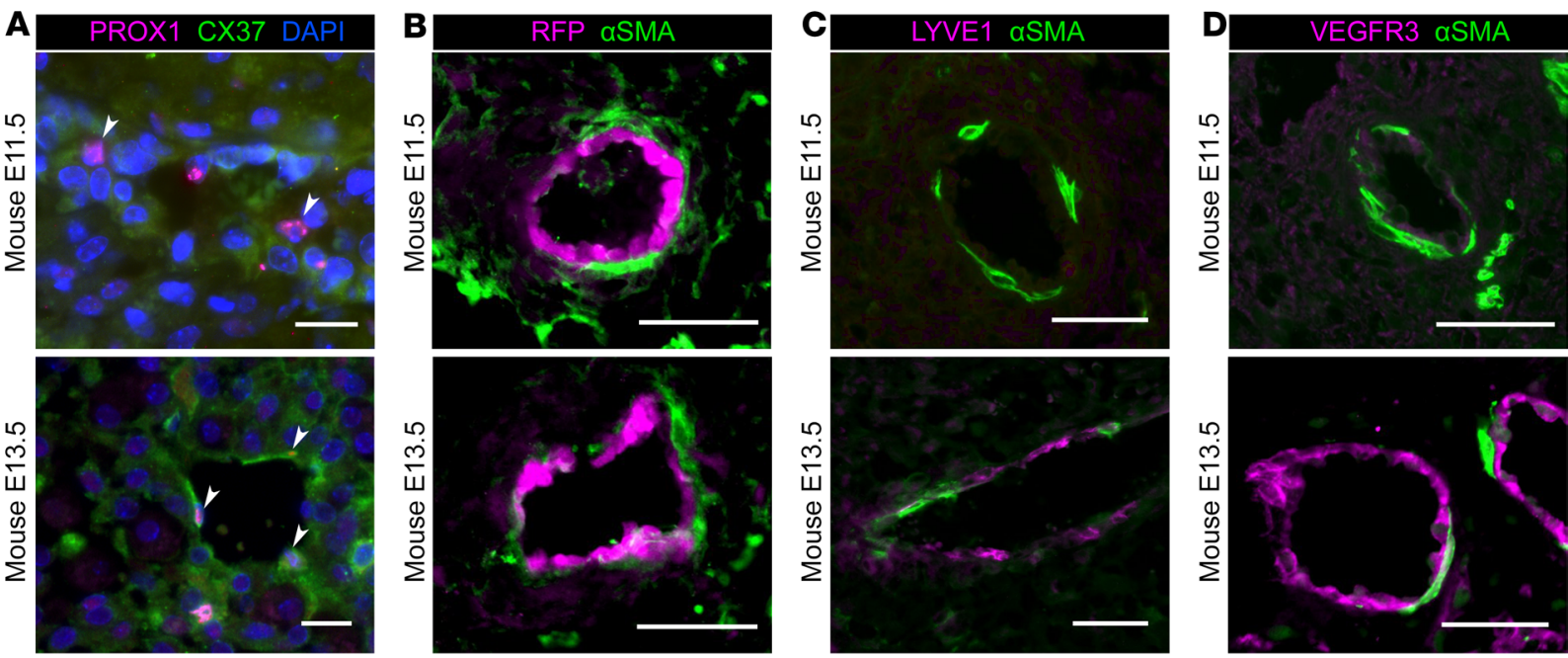

$\mathbf{E}$

Rat E11.5

Rat E13.5
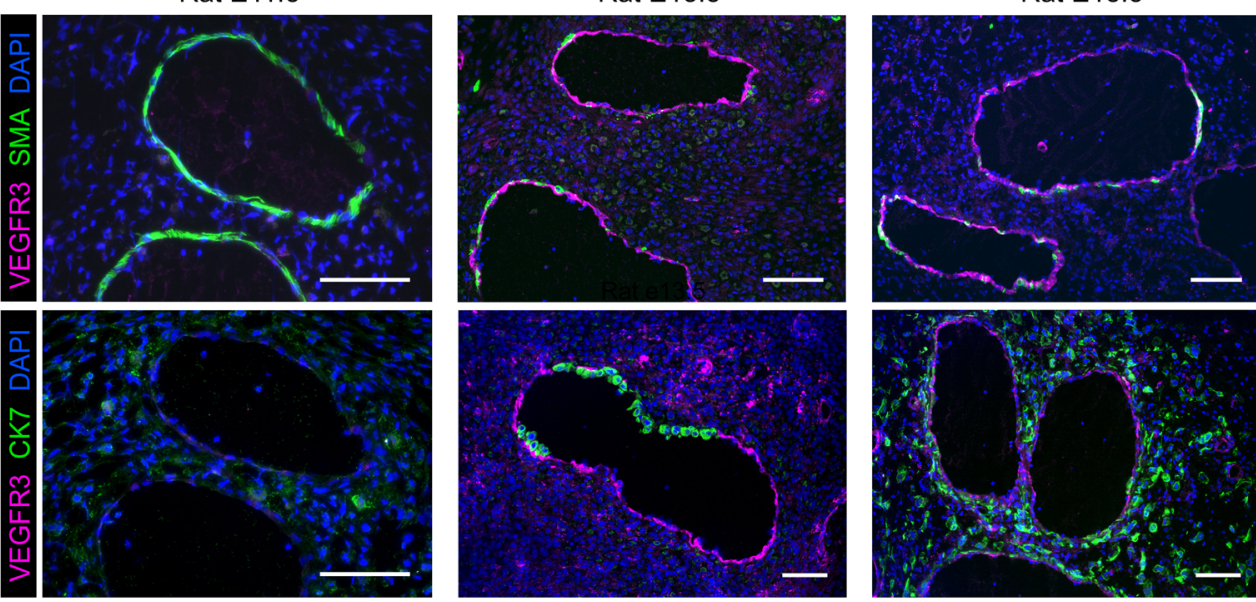

$\mathbf{F}$

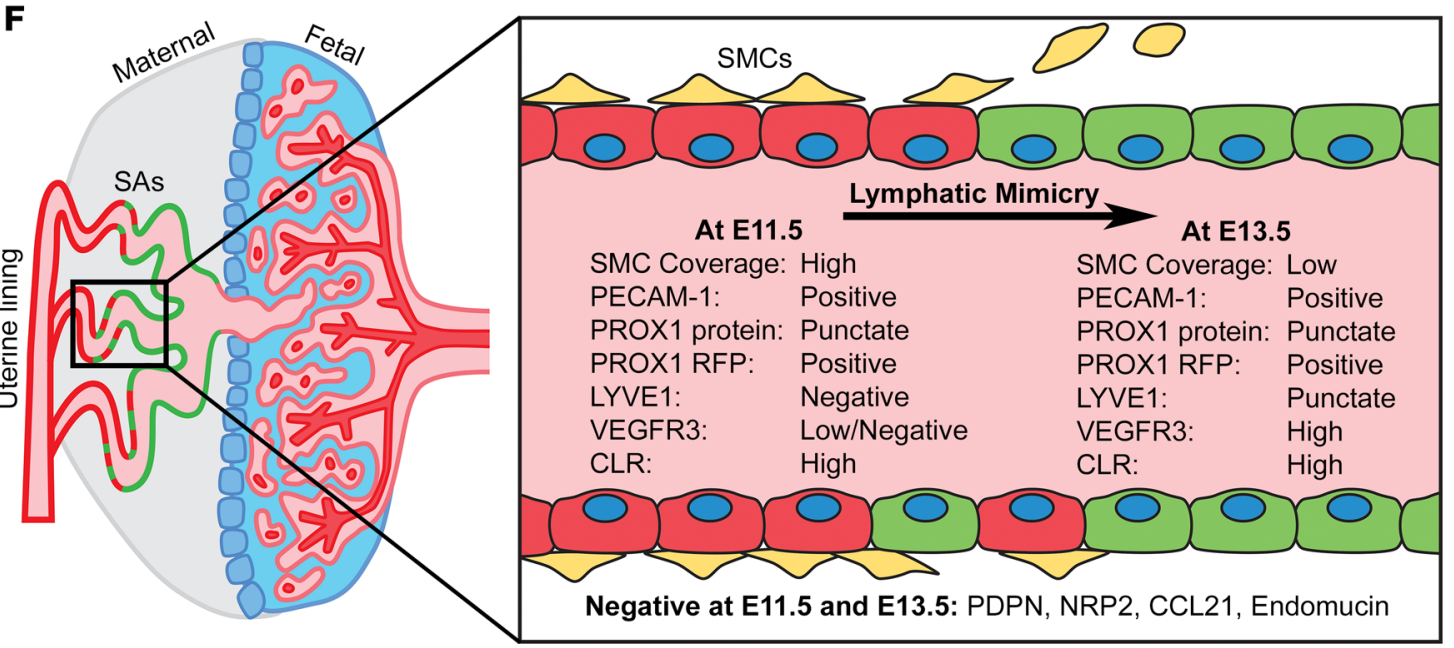

Figure 1. SAs acquire lymphatic characteristics during SAR. (A) Tissue sections of mouse placenta at time points before and after SAR. PROX1 is sporadically expressed at low levels on the SA endothelium at E11.5 and E13.5 (per embryonic day, $n=7-9$ total placentas from 3 litters, with 1-4 placentas from each litter). White arrowheads mark PROX1+ nuclei. Scale bars: $20 \mu \mathrm{m}$. (B) PROX1-RFP+ SA endothelium at E11.5 and E13.5. Scale bars: $50 \mu \mathrm{m}$. (C and D) LYVE1 and VEGFR3 expression is low or absent in SAs at E11.5, but are highly expressed at E13.5 (per embryonic day, $n=8-12$ total placentas from 3-4 litters, with 2-4 placentas from each litter). Scale bars: $50 \mu \mathrm{m}$. (E) Tissue sections of rat placenta at E11.5 show absent VEGFR3 expression while at E13.5 and E18.5 there is robust VEGFR3 expression in SAs. Cytokeratin $7^{+}$(CK7) invasive trophoblasts do not express VEGFR3 (per embryonic day, $n=4-6$ total placentas from 3 litters, with 1-2 placentas from each litter). Scale bars: $100 \mu \mathrm{m}$. (F) A model summarizing features of lymphatic mimicry in SAs during remodeling. SMC, smooth muscle cell. 
calcitonin receptor-like receptor (CLR), receptors for the potent lymphangiogenic factors VEGFC and adrenomedullin (AM), respectively (17-19). And although some studies have concluded that the mouse placenta does not contain classical lymphatic vessels $(20,21)$, the high placental expression and requirement of these lymphangiogenic factors during $\operatorname{SAR}(19,22)$ prompted us to ask whether SAs initiate an intraendothelial transition toward lymphatic fate as a mechanism to promote remodeling.

\section{Results}

SAs acquire expression of a subset of lymphatic markers during $S A R$. Remodeled SAs have remarkable similarities to lymphatic vessels, including reduced smooth muscle cell (SMC) coverage, lack of a basement membrane, and a large and dilated lumen, permitting low-resistance, high-capacitance flow of oxygenated blood to the placenta. This prompted us to question whether SAs might adopt lymphatic identity characteristics during remodeling. Using immunohistochemistry to identify lymphatic markers in SAs of mouse and rat placentas, we found punctate PROX1 expression in mouse SA endothelium prior to SAR at E11.5 and after SAR at E13.5 (Figure 1A). Using a reporter mouse expressing red fluorescent protein (RFP) under the Prox1 promoter, Tg(Prox1-tdTomato) $12 \mathrm{Nrud} / \mathrm{J}$, we observed robust RFP expression in SAs at E11.5 and E13.5 (Figure 1B), though the relative expression level was lower than that observed in bona fide lymphatic vessels of the adjacent myometrium (Supplemental Figure 1A; supplemental material available online with this article; https://doi.org/10.1172/ JCI120446DS1). Interestingly, LYVE1 was absent at E11.5, but induced during the remodeling phase at E13.5 (Figure 1C). Given that LYVE1 is also expressed in some macrophages, we ruled out the possibility that these LYVE1 ${ }^{+}$cells may be myeloid derived (Supplemental Figure 1, B and C).

VEGFR3 expression was also low or absent in SAs at E11.5, but then robustly induced by E13.5 throughout the SA endothelium (Figure 1D). Consistently, VEGFR3 expression was concomitant with remodeled regions of the SA, as evidenced by reduced SMC coverage determined by smooth muscle actin ( $\alpha$ SMA) staining (Figure 1, B-D, and Supplemental Figure 1D). VEGFR3 was also detected in the vascular zone, sometimes referred to as vascular sinuses, of the decidua as previously reported (Supplemental Figure 1E and ref. 23). Some lymphatic markers were not detected in SAs, notably NRP2, PDPN, CCL21, and endomucin (Supplemental Figure 2, A-D), while PECAM-1 expression was detected from E11.5 to E13.5 (Supplemental Figure 2E).

In humans and rats, but less so in mice, endovascular trophoblast invasion is a prominent feature of SAR. Thus, we also characterized SAs from rat placentas where remodeling events occur on a similar gestational timescale to mice. As observed in mice, we identified the initiation of prominent VEGFR3 expression between E11.5 to E13.5 which was concomitant with SMC loss, and the expression was maintained through to E18.5 (Figure $1 \mathrm{E}$ ). Invading trophoblasts (cytokeratin $7^{+}$), which can invade intraluminally as early as E11.5 and interstitially from between E14.5 to E18.5 in rats, were not a source of VEGFR3 expression in rat SAs, as evident by a lack of colocalized expression (Figure $1 \mathrm{E})$. As observed in mice, the vascular zone in rats also expresses VEGFR3 (Supplemental Figure 2F). In contrast to mouse, rat SAs did not express LYVE1 (Supplemental Figure 2F), illustrating species variation in vessel heterogeneity. Taken together, these data demonstrate that SAs acquire a hybrid vessel identity during SAR, expressing both blood EC and lymphatic EC markers (Figure 1F), similar to Schlemm's canal $(5,6)$.

Mice deficient in $u N K$ cells lack p-ERK signaling in VEGFR3expressing SA endothelial cells. Many studies on SAR have focused on how the local interstitial cells, such as uNK cells, promote the remodeling process. Indeed, mice lacking uNK cells have impaired SAR, retaining SA SMCs and thickened vessels walls (24). uNK cells secrete matrix metalloproteases and endothelial growth factors, including vascular endothelial growth factor C (VEGFC), a key driver of lymphangiogenesis that signals through VEGFR3 (17, $25,26)$. Since we have found the expression of endothelial VEGFR3 in SAs to be coincident with the loss of SMC coverage, we hypothesized that UNK cell secretion of VEGFC may be contributing to the removal of SMCs during SAR. Thus, we utilized $I l 2 r \gamma^{t m l W j l}$ mice which are devoid of uNK cells (24) and evaluated their SA endothelial cells for p-ERK as an indicator of endothelial activation. First, we confirmed that $I l 2 r \gamma^{t m 1 W j l}$ mice lacked uNK cells in the mesometrial decidua (Figure 2A) and retained aberrant SMC coverage at E13.5 (Figure 2B). Despite this SMC retention, we found that the SAs of $I l 2 r \gamma^{\text {tm }}{ }^{2 W l}$ placentas still upregulated VEGFR3 level at E13.5, compared with controls (Figure 2B). Congruent with the timing of SAR and absence of VEGFR3 expression, we found low levels of p-ERK in the SA endothelium of both WT and $I l 2 r \gamma^{\text {tmlwjl }}$ mice at E11.5 (Figure 2C). However, while WT VEGFR3-expressing SAs showed a robust increase in p-ERK staining at E13.5, there was no significant increase in $I l 2 r \gamma^{\text {tmIWj }}$ SAs (Figure 2, C and D) despite similar VEGFR3 expression (Figure 2E), demonstrating that the absence of uNK cells abrogates signaling within VEGFR3-expressing endothelial cells. Until now, the main function of uNK cells during SAR was thought to be related to extracellular matrix degradation and disruption of vessel wall SMCs (25). These data reveal that uNK cells also communicate with the endothelium, which promotes ERK signaling, likely in part through VEGFR3, but could also be coincident with other activated pathways.

Mice with kinase-dead VEGFR3 poorly remodel their spiral arteries and exhibit fetal growth restriction. To directly assess the role of VEGFR3 during SAR, we analyzed SAs of heterozygous Flt4hy/+ mice, which carry a dominant-negative, kinase-dead mutation, effectively blocking ligand-mediated receptor autophosphorylation $(27,28)$. Homozygous animals for this Flt4 (also known as Vegfr3) point mutation show complete lack of lymphatics and die immediately after birth due to respiratory failure, but the heterozygotes survive with some lymphatic deficiencies, including chylous ascites from which chy is derived (29). We first confirmed that there was no significant difference in uNK cell number or density in the decidua of Flt $4^{\mathrm{Chy} / \mathrm{+}}$ mice compared with WT mice (Figure 3, A and B), and that VEGFR3 was still expressed in Flt ${ }^{\mathrm{Chy} /+}$ SAs, similar to WT SAs (Figure 3C). To determine whether endothelial signaling is disrupted like in $I l 2 r \gamma^{t m 1 W_{j} l}$ SAs, we examined endothelial ERK phosphorylation and found Flt $4^{\mathrm{Chy} /+}$ SAs to have blunted ERK phosphorylation at E13.5 compared with WT controls (Figure 3D). To assess spiral artery remodeling, we next compared the SMC coverage, luminal area, and vessel wall thickness of Flt $4^{\mathrm{Chy} /++}$ SAs to WT SAs. While no significant difference was observed before SAR 
$\mathbf{A}$

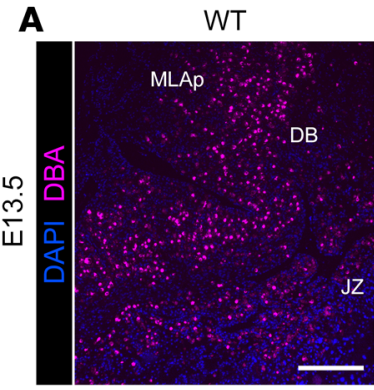

B
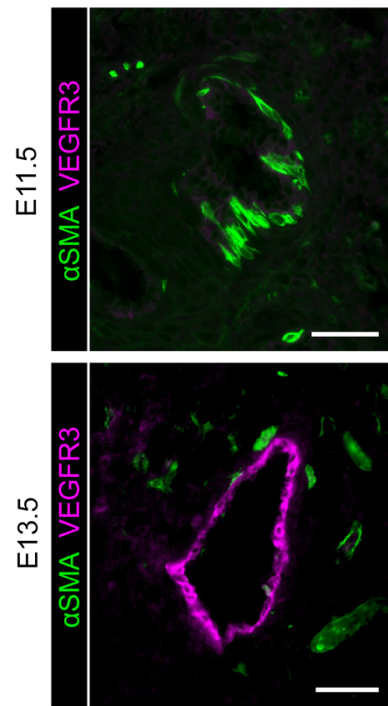

D

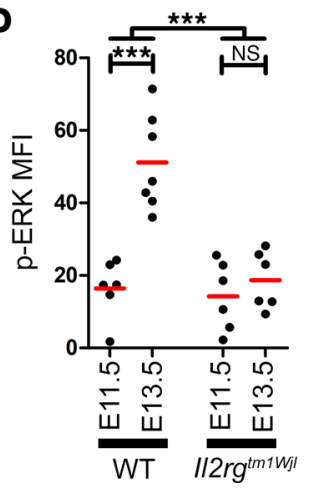

$\| 2 \operatorname{rg}^{\text {tmitwi }}$

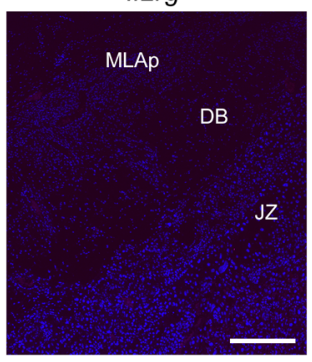

$112 \mathrm{rg}^{\text {tm } \uparrow w_{j} \mid}$
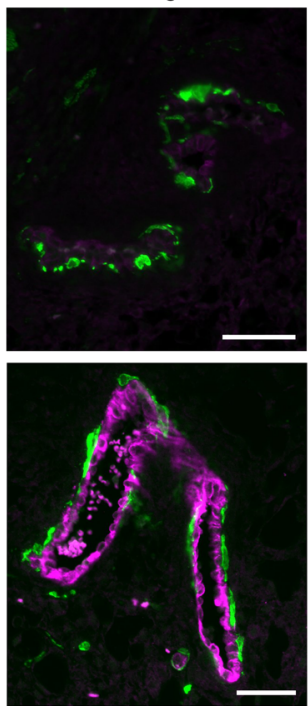

E

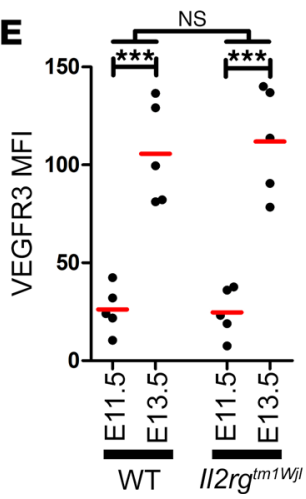

C
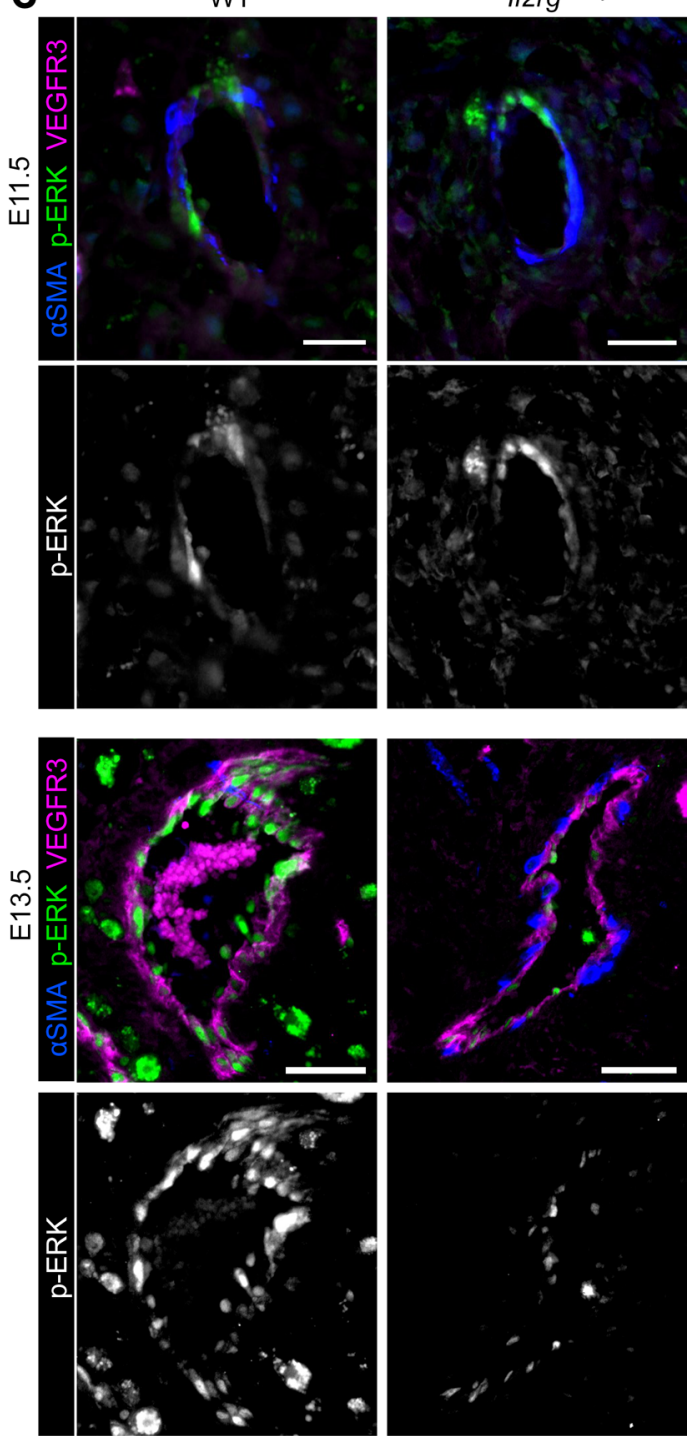

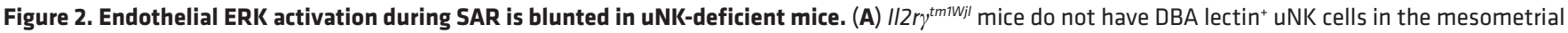
lymphoid aggregate of pregnancy (MLAp) and decidua basalis (DB) (per group, $n=6$ total placentas from 3 litters with 2 placentas from each litter). JZ, junctional zone. Scale bars: $300 \mu \mathrm{m}$. (B) WT SAs exhibit increased VEGFR3 expression and loss of smooth muscle cells from E11.5 to E13.5. $1 / 2 \gamma^{\text {tmiwil }}$ SAs have VEGFR3 expression at E13.5, but SMC coverage remains high. Scale bars: $50 \mu \mathrm{m}$. (C) p-ERK staining increases in the SA endothelium from E11.5 to E13.5 in WT, but not in II2r $\gamma^{\text {tmiWil }}$ mice. Scale bars: $50 \mu \mathrm{m}$. (D) Quantification of p-ERK and (E) VEGFR3 mean fluorescence intensity (MFI) in the SA endo-

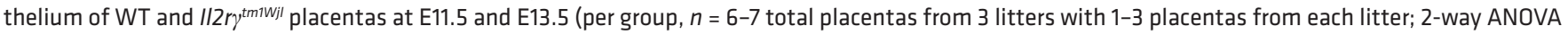
with Bonferroni posttest, $P<0.0001$ for $\mathbf{D}$ and $\mathbf{E}$ ). In all graphs, the red horizontal line represents the mean. ${ }^{* * *} P<0.001$ versus control.

(at E11.5), by E13.5 Flt4 ${ }^{\mathrm{Chy} / \mathrm{+}}$ SAs retained significantly more SMC coverage and failed to undergo the typical increase in luminal area characteristic of SAR and observed in WT SAs (Figure 3, E-G). Furthermore, histological and morphometric analysis revealed that the walls of $\mathrm{Flt}^{\mathrm{Chy} /+}$ SAs remained significantly thickened at E13.5 compared with WT SAs (Figure 3, F and H), likely due in part to the SMC retention, indicating incomplete vessel remodeling. Together, these data establish VEGFR3 signaling as a necessary pathway for promoting spiral artery remodeling.
We next sought to determine if the Flt $4^{\mathrm{Chy} / \mathrm{+}}$ mice exhibit symptoms of preeclampsia during pregnancy. It is established that impaired SAR alone is insufficient to induce symptoms of preeclampsia in mice (30). However, other models of preeclampsia have previously been established in mice (31-33), and additional unknown factors could be at play in Flt $4^{\mathrm{Chy} /+}$ mice. So, we evaluated multiple symptoms of preeclampsia including hypertension, glomerular defects, proteinuria, and fetal growth restriction. We found no significant differences in blood pressure from E15.5 to 

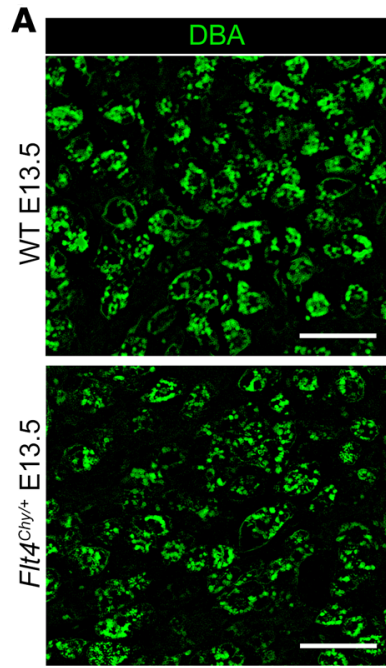

D

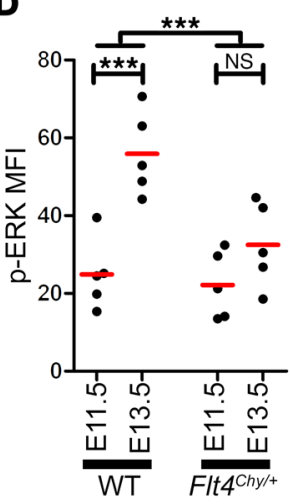

G

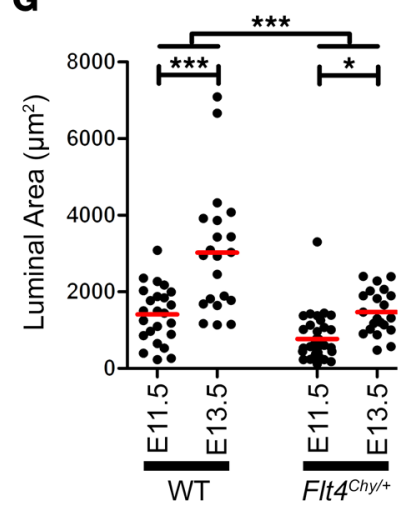

B

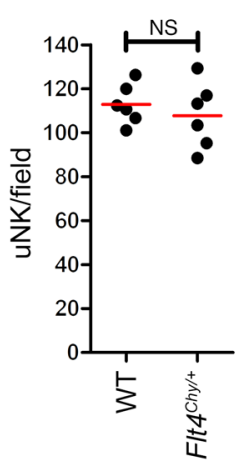

C
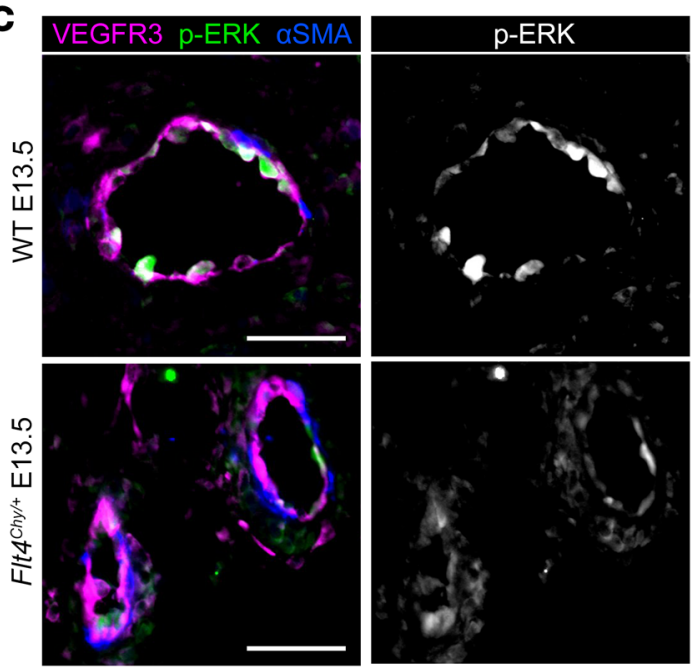

F

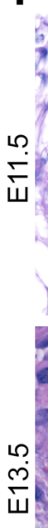

WT

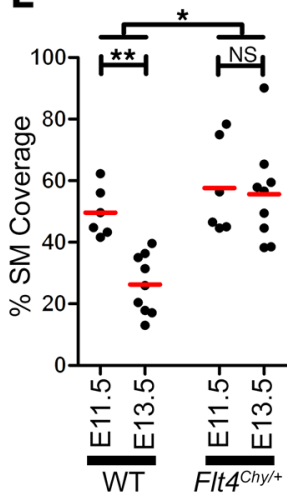

Flt4hy/t+

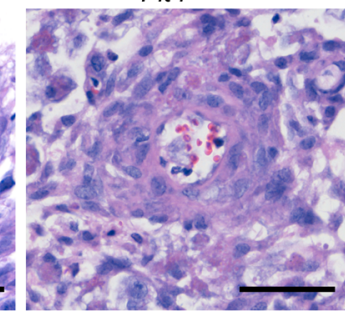

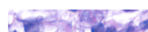
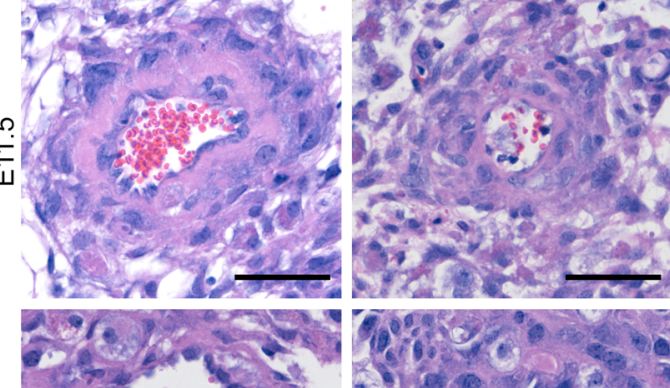

H

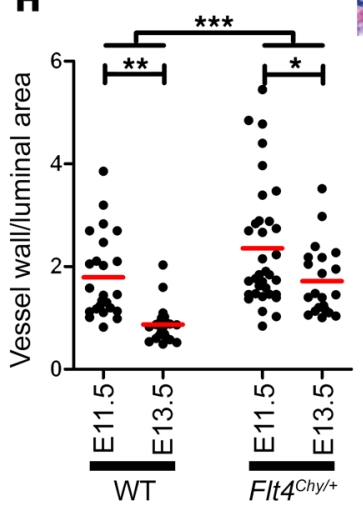

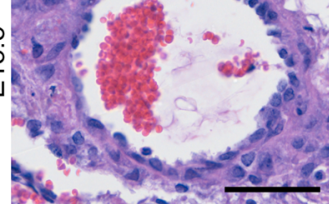
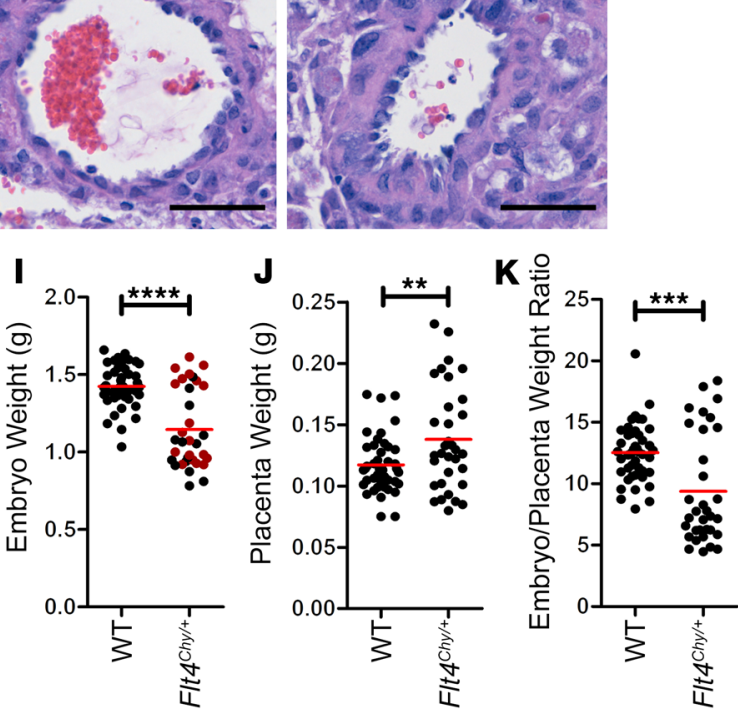

-WT embryo

- FLT4 $4^{\text {Chy/+ }}$ embryo

Figure 3. Loss of VEGFR3 signaling is sufficient to impair SAR. (A and B) The density of uNK cells in the placental decidua of Flt4 ${ }^{\text {chy/+ }}$ mice is similar to WT at E13.5 (per group, $n=6$ total placentas from 3 litters with 2 placentas from each litter; unpaired $t$ test). (C) VEGFR3 signaling-deficient Flt4 ${ }^{\text {Chy/ }+}$ mice retain more SA SMC coverage (blue) compared with WT, even while total expression of VEGFR3 is unchanged. (D) Quantification of endothelial ERK phosphorylation (p-ERK) of WT and Flt4 ${ }^{\text {Chy/+ }}$ mice at E13.5 (per group, $n=5$ total placentas from 3 litters with 1-2 placentas from each litter; 2 -way ANOVA with Bonferroni posttest, $P=0.0002$ ). (E) Quantification of the percentage of SA perimeter covered in $\alpha$ SM-actin ${ }^{+}$SMCs of WT and Flt4 ${ }^{\text {Chy } /+}$ mice at E13.5 (per group, $n=6-9$ total placentas from 3 litters with 2-3 placentas from each litter; 2 -way ANOVA with Bonferroni posttest, $P=0.0001$ ). (F) H\&E staining of SAs in WT and Flt4 ${ }^{\text {Chy/+ }}$ mice at E11.5 and E13.5. (G and H) Quantification of the luminal area and wall thickness (ratio of vessel wall to lumen area) of SAs in WT and Flt4 ${ }^{\text {Chy/+ }}$ mice at E11.5 and E13.5 (per group, $n=21-35$ total placentas from 3 litters with 4-13 placentas from each litter; 2-way ANOVA with Bonferroni posttest, $P<0.0001$ for $\mathbf{G}$ and $\mathbf{H}$ ). (I-K) Flt4 ${ }^{\text {chy/+ }}$ mice exhibit fetal growth restriction and increased placental weights at E18.5 (per genotype, $n=$ 33-44 total embryos and placentas from 3 litters with 9-16 embryos from each litter; unpaired $t$ test). All scale bars: $50 \mu m$. In all graphs the red horizontal line represents the mean. ${ }^{*} P<0.05$; ${ }^{* *} P<0.01$; ${ }^{* *} P<0.001 ;{ }^{* * *} P<0.0001$ versus control. 

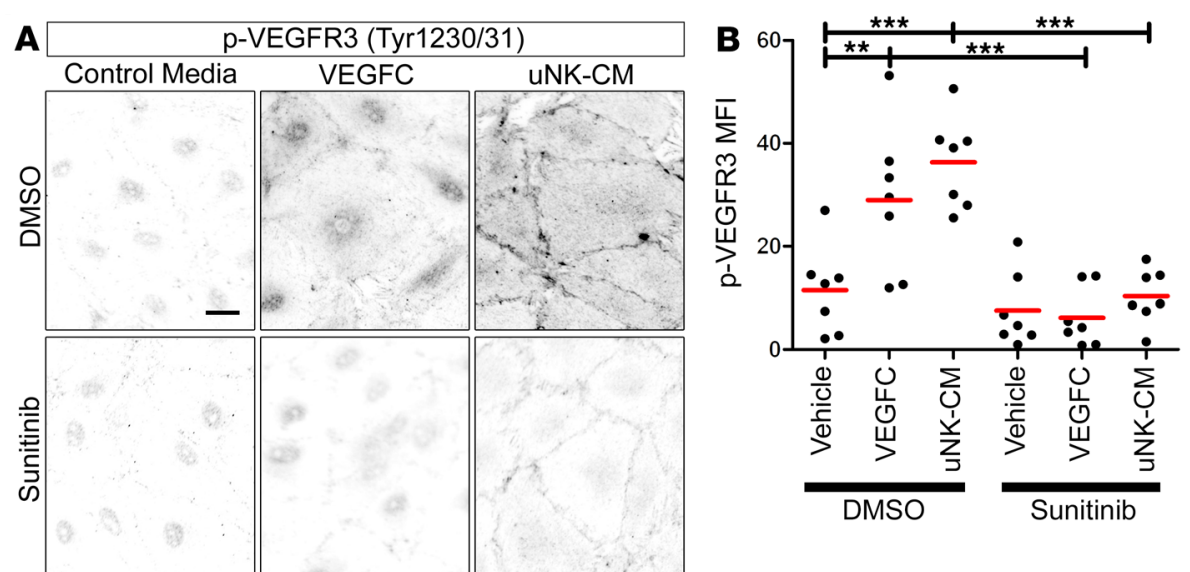

Figure 4. Conditioned media from uNK cells activates VEGFR3. (A) Representative images of $p$-VEGFR3 staining in treated LECs. Scale bar: $20 \mu \mathrm{m}$. (B) Quantification of p-VEGFR3 MFI in LECs of each treatment group $(n=7$ experiments, 1-way ANOVA with Bonferroni posttest). The red horizontal line represents the mean. ${ }^{* *} P<0.01 ;{ }^{* *} P<0.001$ versus control.
E18.5 between Flt4 ${ }^{\mathrm{Chy} /+}$ and WT pregnant mice (Supplemental Figure $3 \mathrm{~A}$ ). Nor did we find any indication of renal dysfunction; no difference was observed in kidney weights, glomerulus size, or Bowman's space area at E18.5 (Supplemental Figure 3, B-E). There was also no difference in the urinary protein concentration at E16.5 (Supplemental Figure 3F). These data demonstrate that Flt ${ }^{\text {Chy/+ }}$ mice do not exhibit overt clinical symptoms of preeclampsia. However, when we examined fetal and placental weights we found significant fetal growth restriction and increased placental weights (Figure 3, I-K), entirely consistent with what has been observed in some uNK cell-deficient mouse models and supporting the conclusion that $F l t 4^{\mathrm{Chy} /+}$ mice exhibit pregnancy deficits compatible with insufficient SAR $(34,35)$.

UNK cell-conditioned medium activates VEGFR3. Given that both VEGFR3 and UNK cells contribute to SA SMC regulation during remodeling, and that UNK cells promote downstream endothelial ERK signaling at the time in which VEGFR3 is initially expressed (Figure 2, C and D), we reasoned that uNK cells may be secreting factors that capitalize upon the newly acquired lymphatic mimicry of SAs. Therefore, we generated uNK-conditioned medium (uNK-CM) by isolating and culturing murine uNKs in vitro as previously described (36), and treated human LECs with this uNK$\mathrm{CM}$ to assess VEGFR3 activation. We first confirmed the presence of VEGFC in UNK-CM by ELISA and found an average of $8.919 \mathrm{ng} /$ $\mathrm{mL}$ of VEGFC $( \pm 0.836 \mathrm{ng} / \mathrm{mL})$. We then confirmed that mouse VEGFC does activate human VEGFR3, as indicated by an increase in p-VEGFR3 immunostaining compared with control medium (Figure 4, A and B, and ref. 37). Using a phospho-specific antibody for VEGFR3 at Tyr1230/1231, we found a significant increase in p-VEGFR3 signal in uNK-CM-treated LECs compared with LECs treated with unconditioned control medium (Figure 4, A and B). When uNK-CM-treated LECs were cotreated with sunitinib, an inhibitor of ligand-mediated VEGFR3 autophosphorylation, the p-VEGFR3 signal returned to baseline. These data establish that uNK cells secrete factors that can activate VEGFR3, with VEGFC being the most likely candidate for ligand-mediated autophosphorylation of residues Tyr1230/1231 (38).

Immune cell-derived VEGFC promotes spiral artery remodeling. To determine whether uNK-derived VEGFC is necessary to activate the SA endothelium and promote SAR, we examined SAs in Vegfct/fl Vav1-Cre mice (Supplemental Figure 4), which do not express VEGFC from immune cells. Even though the Cre is immune cell-targeted, we did not find any significant difference in Vegfcllfl Vav1-Cre uNK density in the mesometrial decidua compared with $V e g f c^{f / f l}$ controls (Figure 5, A and B). Similarly to the Flt4 ${ }^{C h y /+}$ SAs, Vegfctlfl Vav1-Cre SAs have increased SMC retention compared with Vegfct/fl SAs at E13.5 (Figure 5, C and D). Furthermore, lumen area was blunted in Vegfcl/fl Vav1-Cre SAs while the relative wall area increased compared with $V e g f^{f l / f l}$ control SAs (Figure 5, E-G). Together, these findings replicate what was observed in Flt ${ }^{\mathrm{Chy} /+}$ SAs at E13.5, supporting that VEGFC is directly communicating with the SA endothelium via direct activation of VEGFR3 to help promote SAR. Notably, the blunted SAR is consistent with what has been observed in immunodeficient mouse lines, suggesting that VEGFC expression is fundamental to the role uNK cells play to promote SAR in the placenta.

\section{Discussion}

In summary, we have identified a novel instance of lymphatic mimicry within the SAs of the decidua, whereby SAs acquire expression of lymphatic markers, particularly VEGFR3, to facilitate the critical process of SAR. Using genetic mouse models (Figure 6A) and in vitro assays, we describe a novel mechanism whereby lymphatic mimicry of SA endothelial cells render them poised to respond to uNK-secreted lymphangiogenic factors to promote vascular remodeling (Figure 6B). uNK-derived VEGFC binding to VEGFR3 in SAs directly activates the receptor, triggering autophosphorylation and downstream signaling, including ERK phosphorylation. Ultimately, this work identifies a novel endothelium-mediated mechanism by which the placenta and maternal immune system promote SAR to meet the essential needs of the developing fetus, and thereby protect the mother and fetus from the pathological origins of preeclampsia.

Our findings show that SAs are adopting lymphatic expression to promote SAR in an act of lymphatic mimicry. The term lymphatic mimicry is often thought of in cases of nonlymphatic endothelial cells exhibiting aberrant lymphatic expression in response to a disease state or diseased tissue such as a cancerous lesion (39). However, a couple examples of programmed postnatal lymphatic mimicry have been identified in vessels described as hybrid in their identity. This dismantled the previously held belief that programmed initiation of endothelial lymphatic identity in vessels is exclusive to the 

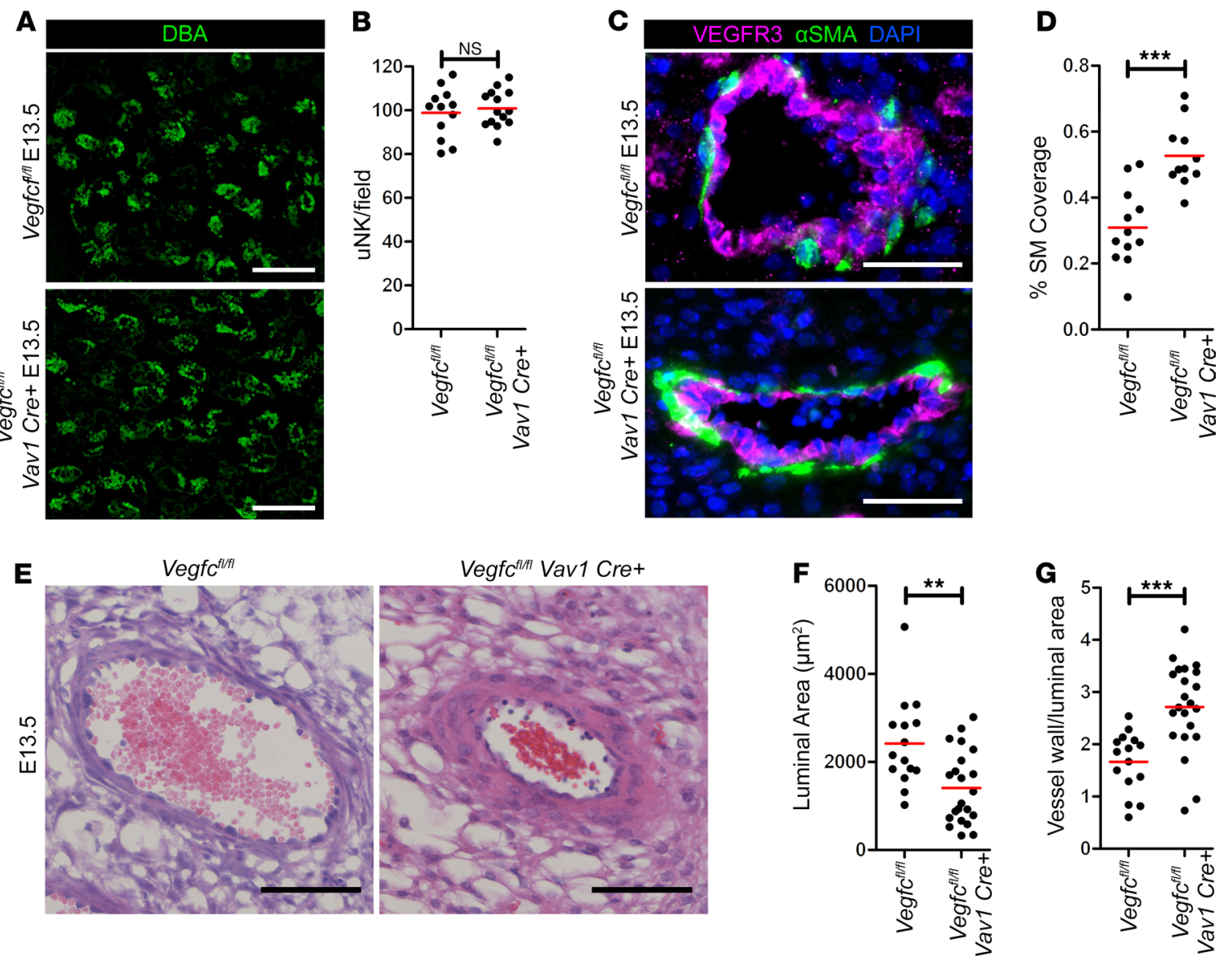

Figure 5. VEGFC from uNK cells is required for SAR. (A and B) The decidua of Vegfc fl/fl Vav1-Cre mouse E13.5 placentas have a similar uNK cell density compared with Vegfc $c^{f / f l}$ placentas as determined by DBA lectin labeling (per genotype, $n=12-14$ total placentas from 3 litters with 2-7 placentas from each litter, unpaired $t$ test). (C and D) At E13.5, Vegff ${ }^{f l f l}$ Vav1-Cre SAs have increased smooth muscle coverage determined by $\alpha$ SMA staining (per genotype, $n=$ 11-12 total placentas from 3 litters with 3-5 placentas from each litter; unpaired $t$ test). (E-C) In H\&E-stained placentas at E13.5, the lumen area of Vegfc $c^{f / f l}$ Vav1-Cre SAs was reduced while the relative wall area was increased (per genotype, $n=15-22$ total placentas from 3 litters with 4-10 placentas from each litter, unpaired $t$ test). All scale bars: $50 \mu \mathrm{m}$. In all graphs the red horizontal line represents the mean. ${ }^{* *} P<0.01 ;{ }^{* *} P<0.001$ versus control.

embryonic development of the lymphatic vasculature. Schlemm's canal of the eye, which is important for draining excess aqueous humor, was first described by 2 independent groups as a hybrid vessel expressing a combination of blood and lymphatic markers $(5,6)$. Additionally, the vasa recta, which runs along the nephron in the kidney to help concentrate urine, was found to express the lymphatic markers PROX1 and VEGFR3 (7). Similar to SAs, these hybrid vessels acquire lymphatic expression to help the vessels perform their highly specialized functions. Unlike the Schlemm's canal and vasa recta, the SAs are not fenestrated nor specialized for fluid homeostasis, and while SAs and the vasa recta are both blood vessels, lymphatic expression in SAs is novel because they are postnatal arteries. This is unusual because lymphatic vessels have historically been considered to have a venous origin, and that a venous identity is the base upon which lymphatic identity is built (40). However, recent work in dermal, mesenteric, and cardiac lymphatic vasculatures prove that not all LECs are venous derived (41). It is possible that the role of lymphatic expression in SAs is distinct from other hybrid vessels because it is influenced by the existing arterial identity. However, it is noteworthy that prior to lymphatic expression, the SAs exhibit a change in the expression of the recep- tor tyrosine kinase EPHB4 and the transmembrane ligand EFNB2, markers of veins and arteries respectively, that indicate a shift toward a venous identity that may be more permissible for the initiation of lymphatic expression (13). Indeed, lymphatic vessels can also express both EPHB4 and EFNB2, and the activation of EFNB2 by EPHB 4 helps facilitate the internalization of activated VEGFR3 to initiate downstream signaling (14). While EFNB2 was found to be expendable for embryonic blood vascular remodeling, it was required for lymphatic remodeling (15). The timing of EFNB2 expression suggests that the SA endothelium may be grooming itself for VEGFR3 signaling just prior to its expression.

By adulthood, VEGFR3 is primarily restricted to lymphatic vessels where it helps lymphatic endothelia grow and maintain their identity in conjunction with PROX1 (42). However, in addition to expression in the vasa recta and other fenestrated endothelia, VEGFR3 has also been implicated in adult blood vesselsprouting angiogenesis (43) and is aberrantly expressed in the proliferating blood vessels of diseased tissue, including tumors $(44,45)$. Furthermore, during development VEGFR3 is widely expressed in fetal blood vessels and is essential for their development $(37,46)$. However, here we show an important role for 
A

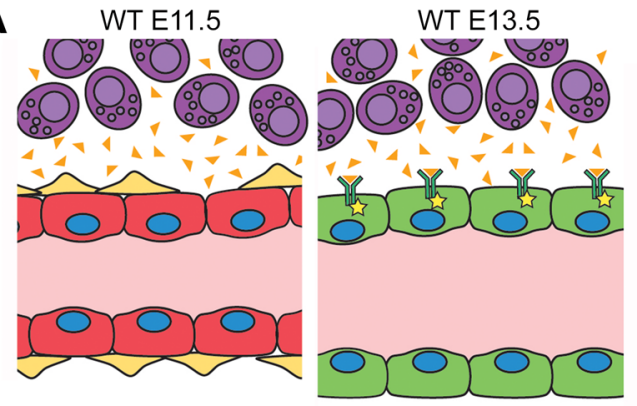

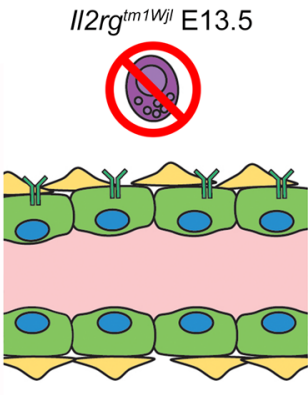
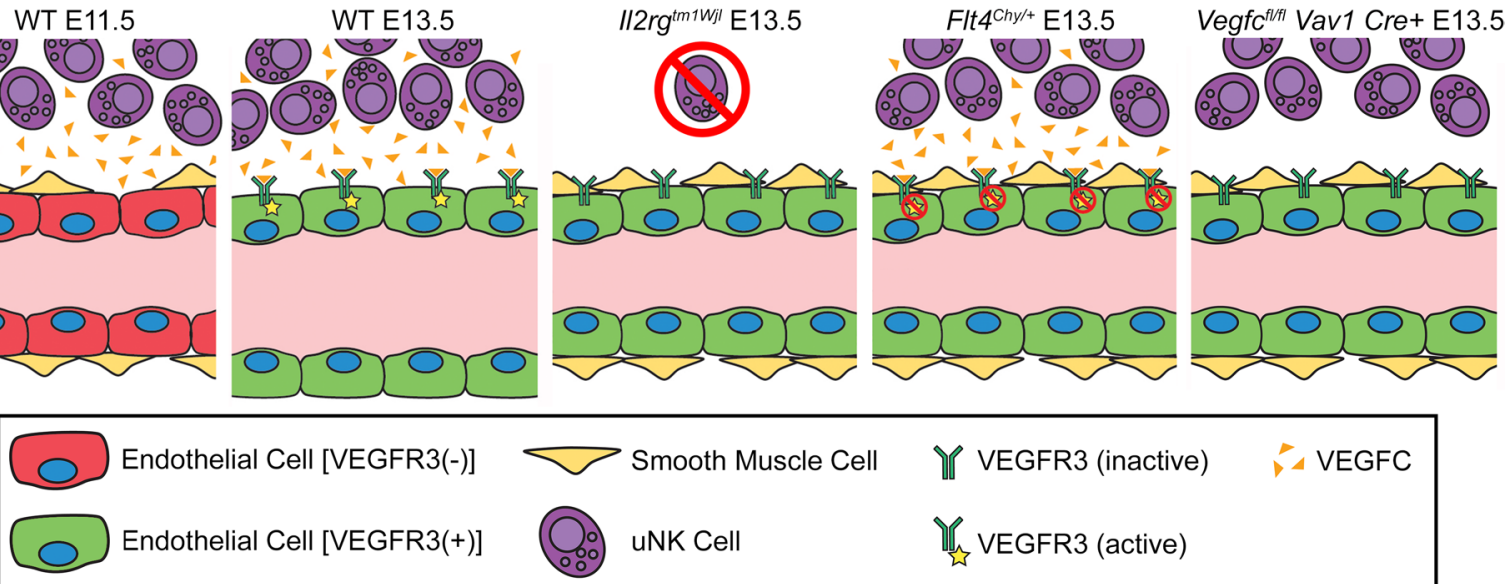

B

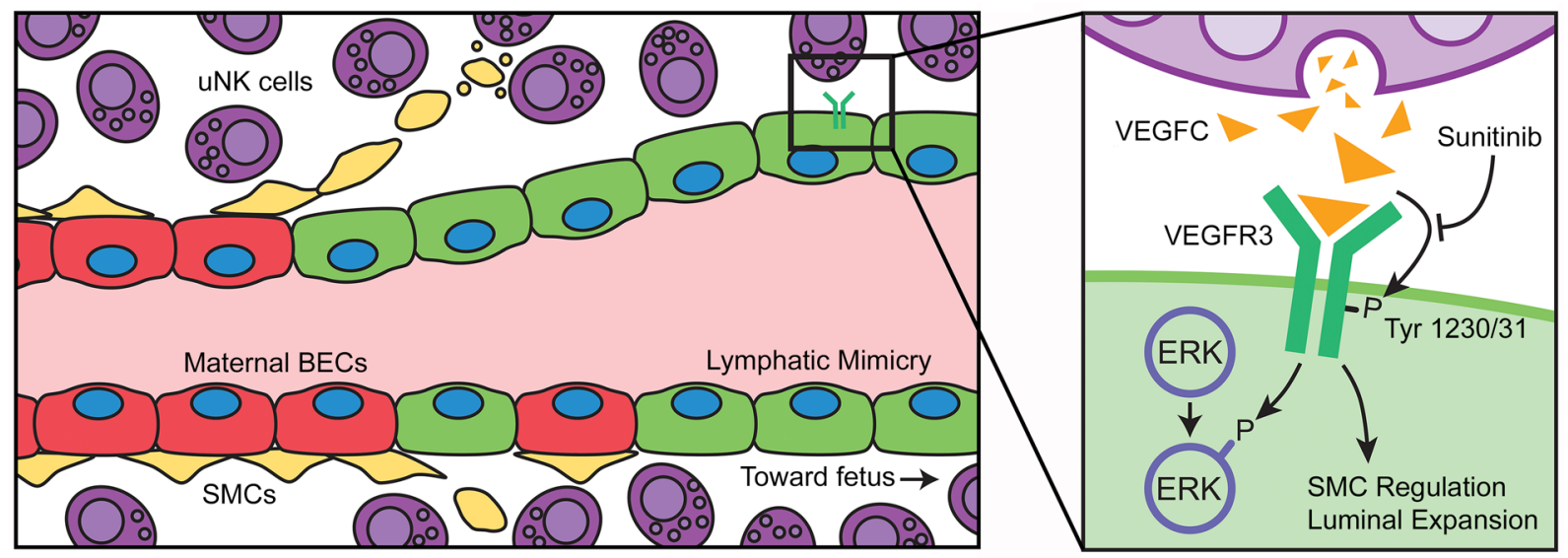

Figure 6. A model of VEGFR3 activation in SAs. (A) Graphical representation of the mechanisms by which the mouse models examined in this study affect VEGFR3 signaling and SAR. (B) Mechanism of Iymphatic mimicry to promote SAR. During SAR, endothelial cells acquire a hybrid vessel phenotype becoming more lymphatic-like; expression of certain lymphatic markers is upregulated, SMC layer is reduced, and luminal expansion occurs.

VEGFR3 in an adult blood vessel where we historically would not have expected to find its expression. We show that VEGFR3 functions as a promotor of vessel remodeling in SAs. Recently, the role VEGFR3 in vascular remodeling has come under the spotlight. It was found that VEGFR3 is responsive to shear stress and that its activation promotes vascular remodeling (47). Also, zebrafish with reduced VEGFR3 expression have a dose-dependent reduction in aorta diameter (47). This is attributed to the association of VEGFR3 with the VE-cadherin mechanosensory complex at cell-cell junctions that activates by a VEGFC-independent, c-Srcmediated mechanism $(48,49)$. In contrast, we show VEGFR3 activation in SAR is VEGFC-dependent, but we suspect the process to be multifactorial and that other factors may make some VEGFC-independent contributions. For example, uNK cells also secrete ANGPT1, ANGPT2, and IFN- $\gamma$, which can help disrupt the cell integrity of vascular smooth muscle cells, and MMPs that degrade extracellular matrix, potentiating smooth muscle regulation of SAs (25). In particular, IFN- $\gamma$ from uNK cells was found to be necessary for SAR, but it is not yet clear if it functions concurrently or in interaction with VEGFR3 signaling (50). Also, shear stress and extracellular matrix are evolving dynamics in SAs during placental development, so signaling pathways that control these biological processes, such as adrenomedullin-CLR signaling, are likely contributing $(47,49)$. Interestingly, we noted that VEGFR3 phosphorylation in lymphatic ECs treated with uNKCM was enhanced at cell-cell borders (Figure 4A) where VEGFR3 could associate with the VE-cadherin mechanosensory complex. Thus, uNK cells may also be appropriating this complex to help promote remodeling.

Preeclampsia in the mouse does not naturally occur to the extent observed in humans, but mouse models of preeclampsia exist $(31,33)$. However, we found that reduced VEGFR3 activity was not sufficient to induce preeclampsia in mice. Though given the differences between mice and humans, we might not have expected the phenotype to be more severe than that observed in uNK-deficient mice, which are not reported to get preeclampsia. Instead, mice deficient for uNK cells are reported to exhibit fetal growth restriction, same as we observed in Flt4 ${ }^{\mathrm{Chy} / \mathrm{+}}$ pregnancies (Figure 3, I-K). This suggests that, while Flt4 ${ }^{\mathrm{Chy} /+}$ pregnancies may not be a model of preeclampsia, they may be useful as model of defective SAR, which contributes to fetal growth restriction similar to uNK-deficient mice, due to endothelial cell signaling insufficiency rather than immunodeficiency. Interestingly though, women with preeclampsia were found to have reduced endothe- 
lial VEGFR3 expression in their spiral arteries (51), suggesting that VEGFR3 may play an expanded role in human pregnancy not observable in mice.

Some fundamental differences in mouse and human placentas must be taken into consideration. First, in humans there is a robust deep invasion of extravillous trophoblast cells into the decidua that integrate into and supplant maternal endothelium, which occurs far less and more shallow in mice (52). The purpose of this invasion is to manipulate SAs to promote further remodeling; however, the period of remodeling examined in this study precedes when this invasion would occur (53). We found in the rat decidua, where robust trophoblast invasion also occurs (54), VEGFR3 was still robustly expressed in the SA endothelium, which coincided with smooth muscle loss as observed in mice. This is also consistent with reports of VEGFR3 expression in human SAs (51). This suggests that trophoblast invasion is not an evolutionary alternative to the lymphatic mimicry observed in mice. Interestingly, in addition to secreting VEGFC, trophoblasts have been reported to acquire VEGFR3 expression after displacing the SA endothelium in humans (55), but we did not find this to occur in rats. Moreover, the human decidua has lymphatic vessels that are absent in the mouse decidua. While mouse trophoblasts had no effect on lymphangiogenesis, human trophoblasts were found to promote it (21). So it is distinctly possible that human trophoblasts contribute a substantial amount of VEGFC, which could rival uNK cells as the predominant source of VEGFC promoting SAR.

It will remain of interest to identify pregnancy- and preeclampsia-related factors that capitalize upon lymphatic mimicry to drive SAR. For example, our previous work shows that fetal trophoblast-derived adrenomedullin (AM) peptide enhances recruitment of uNK cells to the decidua and promotes SAR (19). Our findings here suggest that $\mathrm{AM}$, which is blunted in women with severe preeclampsia, may effectively be increasing decidual VEGFC via uNK cell recruitment. Coincidentally, AM is also a well-established angiogenic and lymphangiogenic signaling pathway, which is notably enhanced in lymphatic endothelium compared with blood endothelium (56). Additionally, PROX1 is a transcriptional regulator of the AM receptor, CLR (56), which is a shear-stress responsive receptor abundantly expressed in SAs (19). So, it is likely that lymphatic mimicry in SAs provides a convergence point for AM and other lymphatic signaling pathways to protect the developing placenta from the vascular onset of preeclampsia.

\section{Methods}

Please see Supplemental Methods for a detailed explanation of all experimental procedures.

Study approval. All experiments were approved by the IACUC of the University of North Carolina at Chapel Hill.

\section{Author contributions}

JBP contributed to the research study design, designed and conducted experiments, acquired and analyzed data, and wrote the manuscript. LB designed and conducted experiments, acquired and analyzed data. LL generated mice and collected tissue. RBD generated mice and analyzed data. ZB acquired and analyzed data. MJS provided archived rat tissue. GO, WM, and MLK generated the $V e g f c^{f l / f l}$ mice and provided tissue. GO provided Prox1-CreER ${ }^{T 2}$ mice. ZJ contributed to research study design, provided Flt $4^{C h y /+}$ tissue, and analyzed data. KMC designed the research study and analyzed data. All authors reviewed and edited the manuscript.

\section{Acknowledgments}

We thank Erika Wittchen, Brooke Matson, and Wenjing Xu for helpful comments and assistance with manuscript preparation, and Regan Scott for preparation of the rat tissue specimens used in the analysis. This project was supported by NIH grants R01 HD060860 (to KMC), HD020676 (to MJS), HD079363 (to MJS), HL073402 (to GO), HL120872 (to MLK), and F31 HD095585 (to JBP); Kirschstein-NRSA training grant T32 2T32HL069768-16 (to JBP); the Lendület program of the Hungarian Academy of Sciences (LP2014-4/2018 to ZJ); and the National Research, Development and Innovation Office (NVKP_16-2016-1-0039 to ZJ).

Address correspondence to: Kathleen M. Caron, Department of Cell Biology and Physiology, 111 Mason Farm Road, 6312B Medical Biomolecular Research Building, CB\#7545, Chapel Hill, North Carolina 27599-7545, USA. Phone: 919.966.5215; Email: kathleen_caron@med.unc.edu.
1. Red-Horse K, Crawford Y, Shojaei F, Ferrara N. Endothelium-microenvironment interactions in the developing embryo and in the adult. Dev Cell. 2007;12(2):181-194.

2. Aird WC. Endothelial cell heterogeneity. Cold Spring Harb Perspect Med. 2012;2(1):a006429.

3. Johnson NC, et al. Lymphatic endothelial cell identity is reversible and its maintenance requires Prox1 activity. Genes Dev. 2008;22(23):3282-3291.

4. Petrova TV, et al. Lymphatic endothelial reprogramming of vascular endothelial cells by the Prox-1 homeobox transcription factor. EMBOJ. 2002;21(17):4593-4599.

5. Aspelund A, et al. The Schlemm's canal is a VEGF-C/VEGFR-3-responsive lymphatic-like vessel. J Clin Invest. 2014;124(9):3975-3986.

6. Park DY, et al. Lymphatic regulator PROX1 determines Schlemm's canal integrity and identity. J Clin Invest. 2014;124(9):3960-3974.

7. Kenig-Kozlovsky Y, et al. Ascending vasa recta are angiopoietin/Tie2-dependent lymphatic-like vessels. J Am Soc Nephrol. 2018;29(4):1097-1107.

8. Soares MJ, Chakraborty D, Kubota K, Renaud SJ, Rumi MA. Adaptive mechanisms controlling uterine spiral artery remodeling during the establishment of pregnancy. Int J Dev Biol. 2014;58(2-4):247-259.

9. Whitley GS, Cartwright JE. Cellular and molecular regulation of spiral artery remodelling: lessons from the cardiovascular field. Placenta. 2010;31(6):465-474.

10. American College of Obstetricians Gynecologists, Task Force on Hypertension in Pregnancy. Hypertension in pregnancy. Report of the American College of Obstetricians and Gynecologists' Task Force on Hypertension in Pregnancy. Obstet Gynecol. 2013;122(5):1122-1131.

11. Lash GE. Molecular cross-talk at the feto-maternal interface. Cold Spring Harb Perspect Med. 2015;5(12):a023010.

12. Brosens IA, Robertson WB, Dixon HG. The role of the spiral arteries in the pathogenesis of pre-eclampsia. J Pathol. 1970;101(4):Pvi.

13. Zhang J, Dong H, Wang B, Zhu S, Croy BA. Dynamic changes occur in patterns of endometrial EFNB2/EPHB4 expression during the period of spiral arterial modification in mice. Biol Reprod. 2008;79(3):450-458.

14. Wang Y, et al. Ephrin-B2 controls VEGF-induced angiogenesis and lymphangiogenesis. Nature. 2010;465(7297):483-486.

15. Mäkinen T, et al. PDZ interaction site in ephrinB2 is required for the remodeling of lymphatic vasculature. Genes Dev. 2005;19(3):397-410.

16. Martin-Almedina S, et al. EPHB4 kinase-inactivating mutations cause autosomal dominant lymphatic-related hydrops fetalis. J Clin Invest. 2016;126(8):3080-3088.

17. Lash GE, et al. Expression of angiogenic growth factors by uterine natural killer cells during early pregnancy. JLeukoc Biol. 2006;80(3):572-580. 
18. Li XF, et al. Angiogenic growth factor messenger ribonucleic acids in uterine natural killer cells. J Clin Endocrinol Metab. 2001;86(4):1823-1834.

19. Li M, et al. Fetal-derived adrenomedullin mediates the innate immune milieu of the placenta. J Clin Invest. 2013;123(6):2408-2420.

20. Red-Horse K. Lymphatic vessel dynamics in the uterine wall. Placenta. 2008;29 Suppl A:S55-S59.

21. Red-Horse K, et al. Cytotrophoblast induction of arterial apoptosis and lymphangiogenesis in an in vivo model of human placentation. J Clin Invest. 2006;116(10):2643-2652.

22. Zhou Y, Bellingard V, Feng KT, McMaster M, Fisher SJ. Human cytotrophoblasts promote endothelial survival and vascular remodeling through secretion of Ang2, PlGF, and VEGF-C. Dev Biol. 2003;263(1):114-125.

23. Kim M, et al. VEGF-A regulated by progesterone governs uterine angiogenesis and vascular remodelling during pregnancy. EMBO Mol Med. 2013;5(9):1415-1430.

24. Croy BA, et al. Histological studies of gene-ablated mice support important functional roles for natural killer cells in the uterus during pregnancy. JReprod Immunol. 1997;35(2):111-133.

25. Robson A, et al. Uterine natural killer cells initiate spiral artery remodeling in human pregnancy. FASEB J. 2012;26(12):4876-4885.

26. Karkkainen MJ, et al. Vascular endothelial growth factor $\mathrm{C}$ is required for sprouting of the first lymphatic vessels from embryonic veins. Nat Immunol. 2004;5(1):74-80.

27. Irrthum A, Karkkainen MJ, Devriendt K, Alitalo K, Vikkula M. Congenital hereditary lymphedema caused by a mutation that inactivates VEGFR3 tyrosine kinase. Am J Hum Genet. 2000;67(2):295-301.

28. Karkkainen MJ, et al. A model for gene therapy of human hereditary lymphedema. Proc Natl Acad Sci USA. 2001;98(22):12677-12682.

29. Jakus $Z$, et al. Lymphatic function is required prenatally for lung inflation at birth. JExp Med. 2014;211(5):815-826.

30. Burke SD, et al. Spiral arterial remodeling is not essential for normal blood pressure regulation in pregnant mice. Hypertension. 2010;55(3):729-737.

31. Ho L, et al. ELABELA deficiency promotes preeclampsia and cardiovascular malformations in mice. Science. 2017;357(6352):707-713.
32. Singh J, Ahmed A, Girardi G. Role of complement component C1q in the onset of preeclampsia in mice. Hypertension. 2011;58(4):716-724.

33. Davisson RL, et al. Discovery of a spontaneous genetic mouse model of preeclampsia. Hypertension. 2002;39(2 Pt 2):337-342.

34. Guimond MJ, Luross JA, Wang B, Terhorst C, Danial S, Croy BA. Absence of natural killer cells during murine pregnancy is associated with reproductive compromise in TgE26 mice. Biol Reprod. 1997;56(1):169-179.

35. Boulenouar $S$, et al. The residual innate lymphoid cells in NFIL3-deficient mice support suboptima maternal adaptations to pregnancy. Front Immunol. 2016;7:43.

36. Croy BA, Zhang J, Tayade C, Colucci F, Yadi H, Yamada AT. Analysis of uterine natural killer cells in mice. Methods Mol Biol. 2010;612:465-503.

37. Kukk E, et al. VEGF-C receptor binding and pattern of expression with VEGFR-3 suggests a role in lymphatic vascular development. Development. 1996;122(12):3829-3837.

38. Dixelius J, et al. Ligand-induced vascular endothelial growth factor receptor-3 (VEGFR-3) heterodimerization with VEGFR-2 in primary lymphatic endothelial cells regulates tyrosine phosphorylation sites. J Biol Chem. 2003;278(42):40973-40979.

39. Elder AM, et al. Semaphorin 7A promotes macrophage-mediated lymphatic remodeling during postpartum mammary gland involution and in breast cancer. Cancer Res. 2018;78(22):6473-6485.

40. Srinivasan RS, et al. Lineage tracing demonstrates the venous origin of the mammalian lymphatic vasculature. Genes Dev. 2007;21(19):2422-2432.

41. Ulvmar MH, Mäkinen T. Heterogeneity in the lymphatic vascular system and its origin. Cardiovasc Res. 2016;111(4):310-321.

42. Srinivasan RS, et al. The Prox1-Vegfr3 feedback loop maintains the identity and the number of lymphatic endothelial cell progenitors. Genes Dev. 2014;28(19):2175-2187.

43. Witmer AN, et al. VEGFR-3 in adult angiogenesis. J Pathol. 2001;195(4):490-497.

44. Partanen TA, Alitalo K, Miettinen M. Lack of lymphatic vascular specificity of vascular endothelial growth factor receptor 3 in 185 vascular tumors. Cancer. 1999;86(11):2406-2412.

45. Paavonen K, Puolakkainen P, Jussila L, Jahkola $\mathrm{T}$, Alitalo K. Vascular endothelial growth factor receptor-3 in lymphangiogenesis in wound healing. Am J Pathol. 2000;156(5):1499-1504.

46. Dumont DJ, et al. Cardiovascular failure in mouse embryos deficient in VEGF receptor-3. Science. 1998;282(5390):946-949.

47. Baeyens N, et al. Vascular remodeling is governed by a VEGFR3-dependent fluid shear stress set point. Elife. 2015;4.

48. Coon BG, et al. Intramembrane binding of VE-cadherin to VEGFR2 and VEGFR3 assembles the endothelial mechanosensory complex. JCell Biol. 2015;208(7):975-986.

49. Galvagni F, et al. Endothelial cell adhesion to the extracellular matrix induces c-Src-dependent VEGFR-3 phosphorylation without the activation of the receptor intrinsic kinase activity. Circ Res. 2010;106(12):1839-1848.

50. Ashkar AA, Di Santo JP, Croy BA. Interferon gamma contributes to initiation of uterine vascular modification, decidual integrity, and uterine natural killer cell maturation during normal murine pregnancy. J Exp Med. 2000;192(2):259-270.

51. Zozzaro-Smith PE, et al. Whole mount immunofluorescence analysis of placentas from normotensive versus preeclamptic pregnancies. Placenta. 2015;36(11):1310-1317.

52. Pijnenborg R, Bland JM, Robertson WB, Dixon G, Brosens I. The pattern of interstitial trophoblastic invasion of the myometrium in early human pregnancy. Placenta. 1981;2(4):303-316.

53. Pijnenborg R, Bland JM, Robertson WB, Brosens I. Uteroplacental arterial changes related to interstitial trophoblast migration in early human pregnancy. Placenta. 1983;4(4):397-413.

54. Vercruysse L, Caluwaerts S, Luyten C, Pijnenborg R. Interstitial trophoblast invasion in the decidua and mesometrial triangle during the last third of pregnancy in the rat. Placenta. 2006;27(1):22-33.

55. Zhou Y, et al. Vascular endothelial growth factor ligands and receptors that regulate human cytotrophoblast survival are dysregulated in severe preeclampsia and hemolysis, elevated liver enzymes, and low platelets syndrome. $A m J$ Pathol. 2002;160(4):1405-1423.

56. Fritz-Six KL, Dunworth WP, Li M, Caron KM. Adrenomedullin signaling is necessary for murine lymphatic vascular development. J Clin Invest. 2008;118(1):40-50. 